\title{
uPrácticas comunicativas como estrategias para hacer frente a la pobreza y exclusión socialı
}

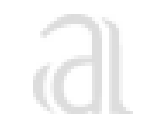

\author{
Paola Bonavitta \\ Recibido: 15 de agosto de 2008 \\ Aprobado: 4 de octubre de 2008
}

\section{Resumen}

Nuestro problema surge frente al fenómeno compartido por toda América Latina de la creciente población pobre que, excluida de la economía productiva, depende de políticas de Estado y de bienes provistos por organizaciones no gubernamentales para su supervivencia. La CEPAL (2001), entre otros, ha reconocido que no todos los pobres están en idénticas condiciones de acceso a bienes debido a su gestión burocrática, uso clientelar, etcétera, lo cual genera nuevas exclusiones y fragmentaciones. Las estrategias tradicionales (públicas y privadas) de lucha contra la pobreza parecen estar en crisis. Sin embargo, recientes investigaciones hacen hincapié en que las redes creadas por los pobres, tanto formales (organizaciones de base) como informales les permiten buscar soluciones a sus propios problemas.

Nuestro trabajo apunta a resaltar la capacidad relacional de los pobres para resolver sus problemas de pobreza y discriminación, dilucidando los mecanismos y recursos sociales, organizacionales, materiales, comunicacionales y simbólicos que se ponen en juego en acciones colectivas en un recorrido histórico específico.

\section{Palabras clave}

Comunicación, capital social, acción colectiva, pobreza, América Latina, redes.

\footnotetext{
* CONICET. Centro de Investigaciones Jurídicas y Sociales
}

Paola_bonavitta@yahoo.com.ar 


\title{
Communication practices as strategies for facing poverty and social exclusion
}

\begin{abstract}
Our problem arises before a common phenomenon in Latin America: growing poor population, which excluded from the productive economy, relies on governmental policies and goods provided by non-governmental organizations for their survival.
\end{abstract}

CEPAL (2001), among others, has accepted that not all the poor people are under the same conditions to access to properties due to the bureaucratic management, corruption, etc., which generates new exclusions and fragmentations.

Traditional strategies (public and private) to fight against poverty seem to be in crisis. However, recent researches focus on the idea that the networks created by poor people, both formal (base organization) and informal networks, help them to look for solutions to their own problems. Our research is aimed to highlight poor people's relational capacity to solve poverty and discrimination problems, finding mechanisms, social, organizational, material, communicational, and symbolic resources which are used in collective actions in a specific historical background.

\section{Key words}

Communication, social capital, collective action, poverty, Latin America, networks. 


\section{INTRODUCCIÓN A LA PROBLEMÁTICA}

Nuestro problema surge frente al fenómeno compartido por toda América Latina de la creciente importancia de la población pobre, que, excluida de la economía productiva, depende de políticas de Estado y de bienes provistos por organizaciones no gubernamentales (ONG) para su supervivencia. La CEPAL (2001), entre otros, ha reconocido que no todos los pobres están en idénticas condiciones de acceso a bienes debido a su gestión burocrática, uso clientelar, etcétera, lo cual genera nuevas exclusiones y fragmentaciones.

Este trabajo se enmarca en un proyecto marco denominado Redes y estrategias contra la pobreza y la discriminación en Córdoba. 19902005 (Valdemarca, Bonavitta y otros, 2005). El mismo ya ha sido finalizado y ha dado origen a un segundo trabajo: Pobreza y satisfacción de necesidades. Estrategias colectivas en Córdoba en la última década (Valdemarca, Bonavitta y otros, 2007). En dichos trabajos hemos intentado analizar la problemática del sostenimiento de la acción colectiva entre sectores populares, mediante las prácticas comunicativas y la acumulación de capital social a través del estudio de caso.

Asimismo, hemos llevado a cabo otra investigación previa, ya finalizada: La comunicación y el sostenimiento de la acción colectiva en Cooperativa Canal de las Cascadas (Bonavitta y otros, 2006). Actualmente, estamos realizando una investigación denominada: Mujeres y capital social: Estrategias para enfrentar la pobreza (Bonavitta, 2008), que forma parte de la tesis doctoral de la autora.
Así, en los trabajos previamente llevados a cabo y en los que actualmente estamos trabajando, hemos podido observar que la pobreza y la discriminación son fenómenos que se han incrementado en América Latina durante las últimas dos décadas. Si bien hay una política de asistencia destinada a los pobres, la gestión burocrática de diagnóstico y distribución, el clientelismo político, etcétera, pueden crear un círculo vicioso donde la mayor necesidad no está acompañada de mayor satisfacción. Los pobres tradicionalmente aseguraron su supervivencia con redes (especialmente basadas en la familia, disponiendo de recursos propios como el trabajo y la vivienda). Pero tales estrategias pueden fallar en contextos de crisis recurrentes.

Sectores importantes de la población dependen cada vez más, para su supervivencia, de políticas de Estado y bienes provistos por organizaciones no gubernamentales, en la medida en que los mecanismos tradicionales generados por los pobres -basados en parentesco y vecindad - pueden perder eficacia en contextos de crisis económicas recurrentes. La capacidad de gestión individual también se resiente por problemas de información de los pobres y de burocratización de los programas, provenientes en general de organismos financieros transnacionales.

En alguna medida, estos elementos pueden actuar de manera discriminatoria condicionando negativamente el acceso de los pobres a los programas, impidiendo mejoras en términos de agencia (desde los pobres) y reduciendo la optimización de asignación (desde los organismos). De este modo, las estrategias tradicionales (públicas 
y privadas) de lucha contra la pobreza parecen estar en crisis. Sin embargo, recientes investigaciones hacen hincapié en que las redes creadas por los pobres, tanto formales (organizaciones de base) como informales, que buscan soluciones a sus propios problemas, pueden ser un factor que rompa este círculo vicioso (mayor necesidad de ayuda que no puede ser aprovechada por las restricciones que la mayor pobreza provoca).

Así, las redes parecen generar estos efectos positivos a través de tres clases de eslabonamientos que los investigadores denominan linking (eslabonamientos hacia delante), bonding (eslabonamientos internos) y bridging (eslabonamientos con organizaciones pares). Asimismo, la existencia de las redes daría a los pobres la oportunidad de participar en las decisiones y dar mayor legitimidad a las resoluciones al permitir la descentralización en la ejecución de políticas.

Nuestro trabajo apunta a resaltar la capacidad relacional de los pobres para resolver sus problemas de pobreza y discriminación, dilucidando los mecanismos y recursos sociales, organizacionales, materiales, comunicacionales y simbólicos que se ponen en juego y se desarrollan en un recorrido histórico específico. Asumimos que la comunicación es la que permite generar redes y capital social entre los pobres, pues es la herramienta que une, organiza y sostiene acciones colectivas entre individuos en situaciones de exclusión y marginalidad, permitiéndoles hacer frente a su contexto.

Siguiendo a Alfaro, consideramos que sin comunicación no es posible el desarrollo. La comunicación permite introducir en el desarrollo una voluntad de compromiso, porque crea vínculos y porque permite que los sujetos involucrados produzcan y construyan sentidos de comprensión de sí mismos, de los demás y de la realidad, posibilitando cambios decididos por los propios sujetos (Alfaro:2006). Si no existe articulación basada en la comunicación y en la confianza no se generarán los apoyos institucionales necesarios ni los intragrupales para poder hacer frente a la situación de exclusión y marginalidad que afecta a gran parte de la población latinoamericana.

Se trata entonces de repensar la creación de capital social en los grupos de pobres y las redes creadas en tanto colectivos generadores de estrategias de lucha contra la pobreza, con la comunicación popular como papel clave. Todo ello se logrará con base en las distintas formas de creación de confianza y conducta cooperativa para que la red se sostenga y logre sus objetivos de gestión, además de la utilización de estrategias materiales y simbólicas de las redes para sostenerse, los vínculos con organizaciones externas -estatales y no estatales- para obtener bienes y establecer si los participantes de las redes perciben su pertenencia como positiva en su lucha contra la pobreza y la discriminación.

El conocimiento de estas formas y mecanismos a través de los cuales se generan y sostienen estas redes es un insumo importante para el diseño de políticas contra la pobreza que pretendan evitar los ya mencionados problemas de ineficacia y discriminación. Estas organizaciones, y la existencia de redes generadas desde el interior de las agrupaciones reunidas en torno a actos 
colectivos, les permiten a los sujetos en situación de exclusión y marginación acceder de manera más eficaz a bienes y servicios.

\section{COMUNICACIÓN Y ACCIÓN}

Si tenemos en cuenta las prácticas comunicativas en el interior de sectores populares, debemos tener presente que adentrarse en lo popular es pensar en identidades frente a alteridades, es tratar de comprender, sobre todo en el caso de la Argentina y de América Latina, cómo logramos una unidad como nación en cada uno de los sujetos que la constituyen desde la pluralidad y la diferencia. Es también contemplar retrospectivamente lo que fuimos y lo que somos, el porqué de la crisis de identidad, la desintegración del concepto de pueblo en múltiples fragmentos. Es repensar el concepto de comunidad e imaginar nuevas formas que permitan avanzar hacia el conocimiento de lo que hoy nos constituye, para posibilitar nuevos procesos sociales que reinventen una sociedad más justa y equitativa y que nos permitan nuevamente recomponer y reconstruir sólidamente nuestra hoy fragmentaria y desvaída identidad.

De esta manera, considerando la vital importancia que las culturas populares tienen en un territorio tan heterogéneo como es Latinoamérica y reafirmando la importancia de estas culturas -que no son subalternas ni secundarias, sino tan válidas como las que tradicionalmente fueron consideradas «hegemónicas»- sostendremos que estos sectores populares son quienes llevan adelante acciones colectivas sostenidas a través del tiempo como la manera para generar lazos y encontrar mejores condiciones de vida. Por otra parte, cabe preguntarnos cuál es el papel que la comunicación y las prácticas comunicativas juegan en el origen, sostenimiento y organización de estas acciones colectivas destinadas a transformar una porción de la realidad social de estos sectores populares.

Además, tendremos en cuenta que sostener estas acciones colectivas significa para los sectores populares que llevan adelante estos actos colectivos transformadores de la realidad social- la posibilidad de crear redes y lazos solidarios entre ellos (intergrupalmente e intragrupalmente) y acrecentar, de esta manera, su capital social, generando una identidad y una memoria colectiva en torno de las acciones colectivas que llevan a cabo.

Las acciones colectivas, como actos destinados a transformar una porción de la realidad social de los sectores populares, son sostenidas y organizadas a través de las distintas prácticas comunicativas y culturales de las personas que las llevan adelante. Estas personas, dueñas de prácticas específicas, son capaces de generar a partir de la creatividad y de la imaginación -sin importar la escasez de recursos en la que se encuentran - posibles soluciones a las dificultades que se les presenten. Sobre todo con el apoyo de otros que, en su misma condición de precariedad y marginalidad, están convencidos de que la mejor manera, y quizá también la más fructífera, de lograr un cambio es través de la unión, la participación y la cooperación entre pares. 
Así, siguiendo a North, las acciones colectivas se logran cuando grupos de individuos enlazados por una identidad en común comparten ciertos objetivos y colaboran entre sí para alcanzarlos, mediante la creación de redes y el trabajo en conjunto (North, 1993). Para que la acción colectiva exista y sea duradera, debe haber una organización, acuerdos, reglas de juego y de conducta que la gobierne, debe haber marcos que posibiliten la comunicación, herramienta que consideramos fundamental para organizar, generar y sostener las acciones colectivas. Estas personas, reunidas en torno a un objetivo en común, generan reglas informales y formales.

La acción colectiva se basa en la cooperación de las personas, en los compromisos asumidos y la confianza generada en el interior del grupo. Pero, para lograr esta cooperación, regulada a partir de ciertas normas y reglas que la equilibren, son necesarias la comunicación y las prácticas comunicativas capaces de generar la coordinación necesaria entre los miembros de la comunidad que se reúnan para formar acciones en conjunto destinadas a cambiar ciertos aspectos de la realidad social en la que se encuentran.

Para otros autores como Tarrow, acción colectiva no es una categoría abstracta que pueda situarse al margen de la historia y de la política (Tarrow, 1997). Coincidimos en ello, puesto que agrupar a la gente en una acción colectiva coordinada en momentos estratégicos de la historia, aún de la historia de la propia comunidad, requiere una solución social, lo cual supone la puesta en escena de desafíos colectivos, la concepción de objetivos comunes, la potenciación de la solidaridad y el mantenimiento del acto co- lectivo. Se lleva a cabo por personas que comparten metas en común y solidaridad en una interacción mantenida con otros grupos o con las elites, los oponentes potenciales y reales y las autoridades.

La acción colectiva es a menudo protagonizada por personas con pocos recursos y escaso poder y, en la base de éstas, se encuentran intereses y valores comunes o solapados entre sí. Estas personas pertenecen a sectores populares, capaces de generar a partir de la creatividad y de sus prácticas culturales y comunicativas un abanico amplio de soluciones sociales ante las adversidades a las que deban enfrentarse (Tarrow, 1997). De esta manera surgieron los cartoneros, los piqueteros, las asambleas barriales, las cooperativas de vivienda y consumo, entre otras, como maneras particulares de hacer frente a las situaciones que los perjudicaban, de enfrentar una realidad que no les resultaba gratificante ni llenaba sus espacios cotidianos. Recurriendo a la organización y a la cooperación, haciendo uso de sus prácticas cotidianas propias de las culturas populares, lograron encontrar la solución a sus problemas y generar, desde sus posibilidades distintas formas de actuar colectivamente.

Así, la magnitud y duración de las acciones colectivas dependen de la organización de la gente a través de las redes sociales y en torno a símbolos identificables extraídos de marcos culturales de significado, es decir, que los involucrados en acciones colectivas necesariamente comparten un conjunto de atributos, por ejemplo: el sexo, la calidad de trabajadores, madres, vecinos, sin tierra, industriales, etcétera. 
Además, toda acción colectiva conforma redes, lo que implica trabajar con otros, formando parte de un proceso donde se intercambia información y recursos, se generan nuevos conocimientos, se potencian las experiencias, se hacen prácticas integradas y se proyecta de forma compartida.

Estas redes contribuyen en la generación de capital social. Además, crean los vínculos afectivos, sociales, relacionales, necesarios para seguir sosteniendo acciones colectivas.

Ante distintas situaciones de precariedad y exposición a diferentes demandas, las acciones colectivas resultan ser la solución idónea, ante todo en los grupos populares, incapaces de generar por sí solos lazos con organismos gubernamentales que pudieran colaborar a mejorar distintas situaciones que los aquejan.

De esta manera, y siendo conscientes de una realidad tan marginal como es la de nuestro país e, incluso, la de Latinoamérica, ante la ausencia de Estados representativos de los intereses de los sectores populares, donde se han agotado el intervencionismo estatal y los canales de representación de la población, la forma más adecuada de hacer frente a las problemáticas diversas que van presentándose es unirse, encontrar la solidaridad y el compromiso de los pares. Ello permite generar marcos y abanicos de soluciones varias que permitan convertir aquello que Marc Augé -desde la antropologíaconcibió como no lugar en un lugar que permita albergar las necesidades, deseos y la posibilidad de presentar la cultura popular y sus prácticas- como una cultura tan válida como la hegemónica.
Por otra parte, tomaremos a Delamata, quien, para el caso específico de acción colectiva en sectores populares, considera que las acciones colectivas «no deben verse solamente como una respuesta desafiante en el marco de una experiencia individual y social machacada por el empobrecimiento, la vulnerabilidad y la exclusión social (...) sino también y específicamente como un acto colectivo transformador de las relaciones sociales (...)» (Delamata: 2004). Las trayectorias de la acción colectiva producen cambios en las relaciones sociales de los sectores populares participantes e introducen nuevos significados a sus vínculos políticos y reposicionan los aspectos instrumentales de la acción social.

Estas acciones, en tanto formas de participación en la vida social, se ponen de manifiesto y adquieren distintas modalidades según el tipo de intervención en la realidad social y, en tanto que son compartidas, son generadoras de nuevas conexiones, ideas y prácticas en el seno del espacio social de sus protagonistas. De este modo, se convierten en un acto colectivo transformador, que permite la modificación de las relaciones sociales en los sectores populares. La acción colectiva supone organizarse en pro de desafíos colectivos para alcanzar objetivos comunes, generar identidad, compartir valores, etcétera.

De esta manera, sostenemos que la comunicación es una de las herramientas que contribuyen a sostener la acción colectiva, porque el lenguaje, medio básico de comunicación entre las personas, amplía las interacciones entre los individuos y los grupos humanos, con lo que se expande la posibilidad de constituirse como sujetos, de compartir marcos y objetivos y de coordinar 
acciones más extensas y complejas mediante redes sociales.

\section{Enredados por la comunicación}

Siguiendo a Alfaro, afirmamos que «sin comunicación no es posible el desarrollo. La comunicación permite introducir en el desarrollo una voluntad de compromiso, porque crea vínculos y porque permite que los sujetos involucrados produzcan y construyan sentidos de comprensión de sí mismos, de los demás y de la realidad, posibilitando cambios decididos por los propios sujetos» (Alfaro, 2006).

Por otro lado, la gestión de los procesos de comunicación en el seno de la organización es una variable fundamental a la hora de favorecer las redes internas de capital social, que permiten una gestión dinámica del conocimiento organizacional.

Las prácticas comunicativas pueden ser caracterizadas como un cuerpo de conocimientos prácticos, basado en tradiciones compartidas y enriquecida por miles de observaciones y experiencias concretas de la práctica cotidiana. La participación en acciones colectivas de los sectores populares es decisiva, ya que, en gran parte, de ello depende que el proyecto conecte «redes de comunicación cotidiana) (Rodríguez Villasante, 1998).

Dentro de las interacciones personales, las representaciones sociales son una perspectiva de análisis específica, una de cuyas funciones es hacer que lo extraño resulte asequible, traduciendo el mundo de lo científico a lo conocido (Moscovici: 1979; Jodelet: 1986; Farr: 1986; Sandoval Moya: 2004). Así, éstas se convierten en un esquema sociocultural de interpretación que permite categorizar lo desconocido.

Estas representaciones se nutren de información a partir de las prácticas comunicativas y de orientaciones globales o predisposiciones, a partir de las prácticas culturales, es decir, tienen su origen en los procesos de interacción de la vida cotidiana. Por tanto, podríamos afirmar que son producto, y a la vez proceso: determinan las percepciones de la realidad, a la vez que son determinadas por ella.

Y es en este contexto en el que se da aquella negociación compleja referida anteriormente, espacio que Moscovici, Jodelet y otros, denominan 'universo consensual'. Una innovación, justamente por el hecho de serlo, se enfrenta a experiencias, informaciones, conocimientos, tradiciones, valores y creencias que los individuos han ido asimilando cotidianamente. Un verdadero sistema de referencia para la toma de decisiones (Moscovici, 1979; Jodelet, 1986; Farr, 1986; Sandoval Moya, 2004).

Las prácticas comunicativas implementadas por sectores populares, desde la creatividad y la intuición y con escasos recursos, redundan en una forma de «empoderamiento» de sus actores.

Entendemos que las interacciones comunicativas que alimentan la vida de las diferentes redes constituyen los entornos simbólicos inmediatos en los que los individuos se reconocen y en los que se refugian sus actitudes respecto al trabajo y a la organización, lo que genera corrientes de opinión muchas veces divergentes e incluso 
contradictorias. Parece plausible afirmar que, desde este punto de vista, la cultura de una organización funciona más como una opinión pública que como una ideología o como una mentalidad común. Tiene sus líderes locales o nodos que focalizan las informaciones, que entretejen en torno a sí opiniones y disposiciones que albergan distintas visiones de su misión, y que filtran informaciones del interior y del exterior con arreglo a sus diferentes historias o aspiraciones.

Las redes intraorganizativas de interacción crean climas de opinión que proporcionan los pseudoentornos en los que se inscriben las lógicas de acción que cada una de ellas impulsa.

Por otro lado, la capacidad de actuación de las organizaciones en términos de su adaptación al entorno no es la suma de las actuaciones de sus integrantes. El conocimiento colectivo no es sólo la agregación de los conocimientos individuales, que deben ser gestionados, esto es, conocidos por los otros y coordinados en función de una estrategia colectiva.

Así, las acciones colectivas que colaboran en la creación de redes se convierten en un auténtico proyecto (es decir, cuando el compromiso con los objetivos de la organización permite hablar de una comunidad). Existe, entre los sectores populares, una determinada motivación que podríamos describir como una motivación comunitaria. Esta estrategia es más eficaz a medida que existe mayor colaboración entre las partes integrantes, y la actitud cooperativa se estimula porque los beneficios que se extraen de ella son percibidos como atractivos.
La gestión de los procesos de comunicación en el seno de la organización es una variable fundamental a la hora de favorecer las redes internas de capital social, que permiten una gestión dinámica del conocimiento organizacional.

Las organizaciones, como las sociedades, se reproducen a través de un proceso comunicativo que se convierte en central en su vida cultural. En ella se entretejen y se negocian las expectativas y las actitudes de los miembros respecto a la organización como la imagen de ésta. La interacción comunicativa no es nunca una secuencia unidireccional. Cuando las organizaciones cooperativas de pobres quieren formar modelos consultivos, participativos o democráticos que potencian la mejora del grupo porque son capaces de gestionar (seleccionar y compartir) un mayor y mejor stock de conocimientos, resultan más eficaces los procesos comunicativos que se conducen en espiral.

Cabe aclarar que la comunicación interna de tipo informal es la más relevante para el análisis de las redes de interacción intraorganizativa y para la traslación del conocimiento tácito.

Es la existencia de una fluida red de comunicaciones en el seno de la organización lo que permite una más eficaz gestión del conocimiento tácito y explícito, pues permite manejar y compartir una mayor cantidad de informaciones heterogéneas. No es sólo tener mayor información sino compartir más lo que ayuda a elegir mejor. De ahí la importancia del logro de esta especie de intranet, que permite la integración y la selección más 
dinámica de las informaciones y de las experiencias más adecuadas.

\section{La comunicación: herramienta fundamental en el sostenimiento de las acciones colectivas}

Como hemos esbozado anteriormente, la comunicación sirve para realizar acciones, para organizarse e intercambiar opiniones dentro de un grupo. Es un vínculo entre grupos humanos, dentro o desde los cuales se relacionan los individuos entre sí y se realiza a través de diversas prácticas comunicativas, que organizan la cooperación entre las personas, la acción de obrar en conjunto para un mismo fin o contribuyendo al éxito de una misma cosa.

Las prácticas comunicativas implican la interacción entre las personas éticamente autónomas, con un reconocimiento del otro, lo que genera un saber compartido, un diálogo. De esa manera, es producción social de sentido, a diferencia de la información, de carácter unilateral, en la que puede haber coacción y no reconocimiento del otro. Consideramos que es imposible no comunicarse, ya que la comunicación es un todo integrado en el que todo comunica: el lenguaje verbal, los silencios, los gestos, el espacio, etcétera. A su vez, la comunicación como fenómeno constitutivo de lo humano y lo social hace posible la construcción, reproducción y transformación de la sociedad.

Siguiendo a Mata y Von Sprecher, entendemos que las prácticas comunicativas

(...) son una de las dimensiones fundamentales de la construcción de lo social (...) a partir de la comunica- ción se van procesando identidades, normas, valores, se van articulando intereses, se van acumulando y legalizando saberes (...) (Morales: 2001).

Asimismo, el abordaje de la comunicación está abierto a todo aquello que pueda aprehenderse con esta práctica, como los procesos del lenguaje, del gesto, en la expresión e interacción, las estructuras y convenciones humanas. Se piensa la comunicación como ligada a la liberación del habla, a la actividad y creatividad popular. A partir de la apertura de los estudios de comunicación y la designación de esta disciplina como ciencia, se pueden lograr aportes a la sociedad entera, reconociendo la importancia del comunicarse, de la interacción cotidiana, de los rumores que entrecruzan la vida de los sujetos y que logran ir generando lazos, creando redes y vínculos y delinear y resignificar identidades y memorias.

Pensamos, de acuerdo con Saintout, que la comunicación popular está conformada por una heterogeneidad de experiencias, prácticas y teorizaciones (Saintout: 2003). Los protagonistas de la comunicación popular son los sectores populares, entendiéndolos como sujetos de producción y cultura, con una particular manera de ver el mundo que va más allá de la resistencia y la oposición a los dominadores. La comunicación entendida desde una perspectiva cultural, tal como lo plantea Jesús Martín Barbero, es mediadora indispensable para comprender los fenómenos comunicativos (Martín Barbero, 1987).

Los procesos de comunicación implican redes sociales amplias, dinámicas, en constante cambio. Por ello, los trabajos comunitarios permiten la interacción de dis- 
tintos actores con sus intereses y prácticas culturales, lo cual organiza la comunicación hacia el interior del barrio, generando o fortaleciendo las redes locales. Esta comunicación posibilita llevar a cabo una tarea conjunta cuando los sujetos se vinculan con el objetivo común de potenciar la solidaridad colectiva a partir de la unión y la organización en un ámbito en común, de manera natural y espontánea. De este modo, se refuerza el trabajo comunitario y se incrementa la capacidad de acción de los grupos en torno a las necesidades y temas prioritarios.

El impacto de las acciones colectivas llevadas a cabo en estos espacios compartidos y su continuidad en el tiempo dependen de la movilización de la gente en las redes sociales alrededor de los símbolos que conforman sus marcos culturales de significado. Tales marcos no están inmediatamente disponibles sino que requieren de la intervención de un agente para convertirse en marcos de una acción colectiva. Así también, estas redes proporcionan «marcos colectivos de contención socio-afectiva, de inclusión desde donde construir identidades y desarrollar una subjetividad» (Delamata: 2004). Son los valores, las visiones del mundo y del sí mismo, los proyectos, los logros, las frustraciones, etcétera.

Por ello, consideramos que, en el marco de las acciones colectivas, como maneras de abordar distintos conflictos que no puedan solucionarse por medio de agentes externos a los grupos populares, la comu nicación actúa como el punto de partida para generar una metapreferencia por la acción colectiva como forma de alcanzar objetivos, donde todos los partícipes son formalmente iguales, organizados comunitaria y cooperativamente.

Consecuentemente, la tarea de la comunicación y las prácticas comunicativas dentro de acciones colectivas consisten en transformar el orden de preferencia individual en otro (u otros) que, pensando en el beneficio común de todo el grupo, promueva la cooperación. Mediante diversas motivaciones comunicadas al colectivo grupal se jerarquizan sus preferencias, entonces pueden contar con metapreferencias y actuar acorde con las mismas. Los individuos, al transformar su orden de preferencias, actúan de cierta forma con el objetivo de mejorar la situación del grupo, en aras del bienestar colectivo y, por ello, toman parte de acciones colectivas.

Esto es así ante todo si tenemos en cuenta la ausencia de un Estado representativo, de autoridades que defiendan la participación popular y tengan en cuenta las demandas de estas personas para intentar satisfacerlas. Ante las carencias de todo tipo que los sectores populares deben enfrentar cotidianamente, y ante las ausencias de mecanismos gubernamentales que los apoyen y los protejan, la única manera que tienen estos grupos es hallar, por sus propios medios, la forma de generar un cambio, de crear metapreferencias en pos de alcanzar el beneficio colectivo.

En el inicio de toda acción colectiva, se encuentra un grupo menor que se encarga de incentivar al resto sorteando las dificultades que conlleva dejar de lado los intereses individuales en pos de priorizar el beneficio colectivo. A este subgrupo, Aguiar, siguiendo a Marwell y Oliver, lo denomina 
masa crítica, «un subgrupo interesado en suministrar el bien colectivo para el grupos y reducir la sensación de incertidumbre del colectivo (Marwell y Oliver: 1993). Reducir esta incertidumbre es la función principal de las instituciones que generen los actores involucrados en una acción colectiva. Tales instituciones pueden ser formales e informales, estableciendo una estructura estable de la interacción humana.

Asimismo, para sostener estas instituciones, es imprescindible la comunicación: para crear lazos de confianza, redes de solidaridad que contribuyan al sostenimiento de la acción y permitan a los sujetos mejorar su posicionamiento, alcanzar un cierto bienestar, educarse, capacitarse, acumular distintos saberes y capital social. Éste supone relaciones formales e informales -capaces de perdurar en el tiempo- que permiten generar lazos de cooperación y confianza para lograr resolver problemas que afecten al colectivo, además de servir al incremento de otros capitales (culturales, económicos, humanos, etcétera). El hecho de pertenecer a distintas redes mejora las capacidades tanto individuales como grupales y genera condiciones para la acumulación de capital social. El capital social contribuye a la creación de capital humano a partir de que los actores establecen relaciones intencionadamente con otras personas que perduran a lo largo del tiempo cuando siguen proveyéndoles beneficios.

Por otra parte, las prácticas comunicativas son esenciales para cambiar el orden de preferencias de los sujetos y volverlos cooperativistas, implementar normas que sostengan y afiancen la solidaridad y la unidad del grupo, evitar el oportunismo y la deserción de la acción colectiva, en suma, generar condiciones para la creación de capital social.

De esta manera, cabe reconocer, volviendo al punto inicial, que la comunicación permite conformar identidades, normas y valores dentro de la acción colectiva de los sectores populares para articular intereses que prioricen el bienestar del grupo, desde el seno del mismo, para transformar activa y comprometidamente su realidad social. También es necesaria para recuperar el protagonismo, la memoria, los logros, de modo de alentar y sostener la acción colectiva. La comunicación, además, transforma las preferencias de los individuos, como ya se dijo anteriormente. Cuando un grupo de ciudadanos toma parte en alguna acción colectiva traslada su interés del ámbito privado a la esfera pública.

Según Hirschman, en nuestras sociedades se oscila entre períodos de intensa preocupación por los problemas públicos y otros de concentración casi absoluta en las metas del mejoramiento individual y el bienestar privado (Hirschman: 1989). La participación en los asuntos públicos suele surgir cuando en la vida privada se generan insatisfacciones. Para seguir participando en los asuntos públicos, los individuos deben tener confianza en el grupo, ya que ésta contrarresta la inestabilidad y la incertidumbre evitando las deserciones en masa; deben tener también la capacidad de rescatar sus logros, generar espacios de contención, recuperar a través de la memoria grupal la trayectoria del grupo, sus experiencias.

Teniendo en cuenta que en el marco latinoamericano existen políticas 
imperialistas predominantes, que priorizan las culturas de los sectores dominantes y las prácticas que se imponen «desde arriba», es necesario volver la mirada a aquellas culturas que, históricamente, fueron consideradas subalternas, a esas culturas que predominan en los márgenes y que, con base en la creatividad, la imaginación y la necesidad de ser oídas, de hacerse visibles, han conseguido interpretar su realidad y encontrar la manera de transformarla.

\section{Autogestión eficaz}

Por su parte, creemos que la autogestión de bienes por parte de los pobres opera en varios sentidos. El involucrar a los pobres en las tareas de diagnóstico, obtención y distribución de bienes puede redundar positivamente en su percepción individual y social, y en su accionar como sujetos activos que buscan cambiar sus condiciones sociales a la vez que desarrollan capital social. Simultáneamente podrían garantizar al Estado y a las organizaciones no gubernamentales una gestión más eficaz de los recursos.

Por otra parte, Cohen y Arato aseguran que la sociedad civil se ha convertido en el terreno indispensable en el que se reúnen, organizan y movilizan los actores sociales, pese a que sus objetivos sean otros tales como mejorar su situación económica, resolver sus necesidades básicas y hacer frente a las falencias que tiene el Estado moderno (Cohen, Arato, 2001).

Tilly, en esta misma línea, afirma que han nacido nuevas formas de solidaridad, de asociación, de recursos de poder y de modos de conflicto en el terreno de la sociedad civil moderna; y considera estas formas de organización y protesta como «más autónomas» que las reuniones «espontáneas» características del repertorio de acciones del siglo XVIII (Cohen, Arato, 2001).

Por otra parte, consideramos la capacidad relacional de los pobres como un factor central que garantiza obtención y distribución de bienes y servicios destinados a ellos. Los movimientos sociales organizados como acciones colectivas constituyen el elemento dinámico en proceso que podrían convertir en realidad los potenciales positivos de las sociedades civiles modernas (Cohen, Arato: 2001). Así, el tema de la autodefensa de la «sociedad contra el Estado» (y contra la economía de mercado capitalista no regulada) ha sido planteado por varios actores colectivos contemporáneos que luchan por una sociedad civil autónoma y democrática.

\section{Metodología y técnicas de investigación}

Dadas las características del tema a investigar, hemos optado por una mirada cualitativa por considerar que nos permitirá una profundización mayor en el problema y una mejor aproximación al objeto. Este trabajo constará de un estudio de caso comparativo entre cooperativas de barrio urbano marginal de la ciudad de Córdoba, Argentina.

El uso de técnicas etnográficas, como observación y entrevistas en profundidad, nos permitirá un análisis intensivo y profundo que nos proporcionará, asimismo, un mejor conocimiento de las formas de fun- 
cionamiento de la racionalidad humana en el contexto de pobreza y exclusión.

Por tanto, emplearemos técnicas cualitativas con el fin de aprehender las experiencias más destacadas de la vida de una persona a través del análisis de su discurso. A partir de este tipo de técnicas intentaremos comprender cuál es la significación que otorgan a los acontecimientos y a todo lo que ocurre en esos escenarios.

Por otra parte, y teniendo en cuenta que todos los relatos deben ser analizados como fenómenos sociales que ocurren o son moldeados en contextos particulares, complementaremos la técnica de la entrevista con otra: la observación.

Perfeccionaremos, asimismo, la información con encuestas y datos secundarios, que obtuvimos de diferentes documentos escritos. En este caso puntual indagaremos en la documentación existente en el interior y exterior de las organizaciones: sus reglamentos y estatutos internos, actas de asambleas ordinarias y extraordinarias, la Ley de Cooperativas Nacional.

También haremos uso de indicadores macro-económicos y sociales. Esta técnica se constituye en una estrategia complementaria para entrecruzar datos de archivos documentales y estadísticos, investigaciones realizadas sobre el tema, publicaciones, anuarios y las fuentes orales, etcétera.

Estas diferentes fuentes orales y escritas nos permitirán contar con un entramado empírico de envergadura que posibilite la constatación de las hipótesis y la elaboración de informes parciales y finales.

\section{A MODO DE CONCLUSIÓN}

Teniendo en cuenta las prácticas comunicativas y los alcances de estas en grupos cooperativos, habría que enfocar aún más la mirada en estos sectores populares capaces de encabezar actos colectivos transformadores con los escasos recursos que cuentan: imponiendo sus prácticas, haciendo uso de la comunicación como herramienta fundamental y alterando el orden de preferencias a fin de conseguir el bienestar colectivo.

En América Latina, la identidad propia de los sectores populares se manifiesta en sus heterogéneas experiencias y prácticas, en distintas maneras de abordar, de vivir, de enfrentar y solucionar. Así, crean particulares marcos de contención socio-afectivos a partir de los cuales se refuerzan los distintos capitales de una persona.

Entonces, sería interesante pensar en estos sectores populares y en su solidaridad. Si las culturas hegemónicas se han encargado de dominar al resto durante años, quizá ya sea hora de que empiecen a mirar a su alrededor, que aprendan y comprendan a esas culturas «marginales» capaces de solidarizarse, de cooperar y de interpretar las necesidades del conjunto, para crear la manera adecuada de transformar su realidad social.

La preocupación actual por lo popular se inscribe en la necesidad de un avance imprescindible en los criterios de consideración de los diversos fenómenos 
socioculturales. Al mismo tiempo, cualquier respuesta que se intente resulta compleja, dado que hay una profusa y a veces contradictoria tradición de estudios sobre el tema.

Muchas son las preguntas que podemos formularnos, pero ante todo consideramos que repensar lo popular implica, pues, la revisión y puesta en debate de las ideas fundamentales a las que se debe atender: los alcances del término popular, o prácticas comunicacionales populares, los rasgos mediante los cuales es posible su caracterización, su grado y su forma de determinación en lo masivo, el modo de abordaje.

Así, si bien ya desde su misma definición, lo popular siempre ha generado discusión, creemos de suma importancia el defender «lo popular», las prácticas heterogéneas propias de los sectores populares y las distintas maneras de transformar la realidad social. A lo largo del tiempo, estos grupos, desprotegidos y marginados, han logrado apelar a las acciones colectivas para conseguir aquello que no consiguen a través de los agentes externos que, en teoría, están encargados de hacer de nuestros espacios y realidades un lugar mejor.

Repensar estas estrategias de cara a una Posmodernidad que propone a un sujeto individual en la búsqueda de un individualismo positivo (Giddens, 1993) es una opción viable para evitar la crisis del lazo social. Los más desprotegidos del sistema han apelado a la búsqueda de la cohesión social, anhelada históricamente por teóricos modernos como Durkheim (Durkheim: 1967). Si bien la periferia los atrapó, han hecho frente a esa situación a partir de la constitución de acciones colectivas que les brindaron marcos de contención y redes desde donde repensar un futuro que no los encuentre tan aislados.

\section{REFERENCIAS BIBLIOGRÁFICAS}

Aguiar, Fernando (Comp). (1991). La lógica de la cooperación en intereses individuales y acción colectiva. Madrid: Editorial Pablo Iglesias.

Alfaro, Rosa María. (2006). El desarrollo en la agenda pública. Viabilidad del Cambio Social desde Políticas Innovadoras de Comunicación. Roma: Primer Congreso Mundial de Comunicación y Desarrollo. FAO-BM.

Banco Mundial. (2001). Engendering Development. II Series. New York: Oxford University Press.

Barrancos, D. y Goren, N.(2002). De la exclusión a la organización. Buenos Aires: Ciccus.

Bonavitta y otros. (2007). La comunicación y el sostenimiento de la acción colectiva. La Plata: Question Académica, UNL.

Bontempo, Máximo. (2000). Por una intervención a favor del desarrollo. Madrid: Facultad CC Información. Universidad Complutense de Madrid. 
CEPAL. (2000). «Panorama social de América Latina». Santiago de Chile: Naciones Unidas.

CEPAL. (2001). «Capital Social y reducción de la pobreza: en busca de un nuevo paradigma». Chile: Naciones Unidas.

CEPAL. (2007). «Estadísticas para la equidad de género: magnitudes y tendencias en América Latina». Chile: Naciones Unidas.

Coleman, J. (1990). Foundations of Social Theory. Cambridge: Harvard University Press.

Cohen, Jean; Arato, A. (2001). Sociedad Civil y teoría Política. México: Fondo de Cultura Económica.

Delamata, Gabriela. (2004). Los Barrios Desbordados: las organizaciones de desocupados del Gran Buenos Aires. Buenos Aires: Eudeba. Serie Extramuros.

Durkheim, Emile. (1967). De la división del trabajo social. Buenos Aires: Schapire Editor.

__ (1973). La educación moral. Buenos Aires: Schapire Editor.

_- (1974). El suicidio. México: UNAM.

Durston, J. (1999). «Construyendo Capital social comunitario». Revista 69, CEPAL.

Elson, Diane. (1998). «The economic, the political and the domestic: businesses, states and household in the organisation of production». New Political Economy, vol. 3, No. 2.

Enríquez Rozas, R. (2000). «Redes sociales y pobreza. Mitos y realidades». México: La Ventana, No.11.

Fraser, Nancy. (2002). Política feminista en la era del reconocimiento. Una aproximación bidimensional a la justicia de género. Buenos Aires: PRIGEPP - FLACSO.

Giddens, Anthony. (1993). Consecuencias de la Modernidad. Buenos Aires: Amortorrou.

Hirschman, Albert. (1986). Interés privado y acción pública. México: FCE. Serie Economía.

Hirschman, Albert. (1989). Enfoques alternativos sobre la sociedad y el mercado. México: FCE. Economía contemporánea.

Luhmann, N. 1983. Fe y racionalidad en los sistemas. Madrid: Editora Nacional.

Mata, C. y Von Sprecher, R. en Morales, Susana. (2001). Cambio, cultura y comunicación en la dinámica de las organizaciones. Córdoba: Editorial Mimeo. Escuela de Ciencias de la Información. Universidad Nacional de Córdoba.

March, J. G. y Simon, H. (1987). Teoría de la organización. Barcelona: Ariel. 
Martín- Barbero, Jesús. (1987). De los medios a las mediaciones: comunicación, cultura y hegemonía. Barcelona: Gustavo Gili Editor.

Marwell y Oliver. (1993). The Critical Mass in Collective Action. Annual Review of Sociology. Cambridge: Harvard University Press.

Narayan, D. y Woolcock, M. 2000. Capital social: Implicaciones para la teoría, la investigación y las políticas sobre desarrollo. Washington, D.C.: Banco Mundial.

North, Douglass. (1993). Instituciones, cambio institucional y desempeño económico. México: FCC.

Rodríguez Villasante, Tomás. (1998). Cuatro Redes para mejor-vivir. Vol. 1. Del desarrollo local a las redes de mejor-vivir. Buenos Aires: Lumen / Humanitas.

Saintout, Florencia. (2003). Los movimientos sociales. Viejas y nuevas voces. Abrir la Comunicación: tradición y movimiento en el campo académico. La Plata: EPC Educación. Ediciones de Periodismo y Comunicación. Facultad de Periodismo y Comunicación Social. Universidad Nacional de La Plata.

Sautu, Ruth (Comp). (2000). Las mujeres hablan: Consecuencias del ajuste económico en familias de sectores pobres y medios en la Argentina. Buenos Aires: Ediciones Al Margen.

Sen, Amartya. (2000). Social Exclusion: Concept, Application and Scrutiny. Banco Asiático de Desarrollo.

Stiglitz, Joseph. (1998). Toward a New paradigm for Development: Strategies, Policies and processes. Geneve: UNTAD.

Tarrow, Sydney. (1997). Poder en movimiento: los movimientos sociales, la acción colectiva y la política. Madrid: Alianza.

Terrén Eduardo. (2004). Las organizaciones educativas como sistemas de comunicación. Un enfoque micropolítico. Revista Iberoamericana de Educación. Madrid 